\title{
Subthalamic Stimulation Reduces Vowel Space at the Initiation of Sustained Production: Implications for Articulatory Motor Control in Parkinson's Disease
}

\author{
John J. Sidtis ${ }^{\mathrm{a}, \mathrm{b}, *}$, Amy G. Alken ${ }^{\mathrm{a}}$, Michele Tagliati ${ }^{\mathrm{c}}$, Ron Alterman ${ }^{\mathrm{d}}$ \\ and Diana Van Lancker Sidtis ${ }^{\mathrm{a}, \mathrm{e}}$ \\ ${ }^{a}$ Brain and Behavior Laboratory, Geriatrics Division, The Nathan Kline Institute, Orangeburg, NY, USA \\ ${ }^{\mathrm{b}}$ Department of Psychiatry, New York University Langone School of Medicine, New York, NY, USA \\ ${ }^{\mathrm{c}}$ Department of Neurology, Cedars-Sinai Medical Center, Los Angeles, CA, USA \\ ${ }^{\mathrm{d}}$ Neurosurgery, Department of Surgery, Beth Israel Deaconess Medical Center, Boston, MA, USA \\ ${ }^{\mathrm{e}}$ Department of Communicative Sciences and Disorders, NYU Steinhardt School of Culture, Education, \\ and Human Development, New York, NY, USA
}

Accepted 13 February 2016

\begin{abstract}
.
Background: Stimulation of the subthalamic nuclei (STN) is an effective treatment for Parkinson's disease, but complaints of speech difficulties after surgery have been difficult to quantify. Speech measures do not convincingly account for such reports.

Objective: This study examined STN stimulation effects on vowel production, in order to probe whether DBS affects articulatory posturing. The objective was to compare positioning during the initiation phase with the steady prolongation phase by measuring vowel spaces for three "corner" vowels at these two time frames.

Methods: Vowel space was measured over the initial $0.25 \mathrm{sec}$ of sustained productions of high front (/i/), high back (/u/) and low vowels (/a/), and again during a 2 sec segment at the midpoint. Eight right-handed male subjects with bilateral STN stimulation and seven age-matched male controls were studied based on their participation in a larger study that included functional imaging. Mean values: age $=57 \pm 4.6 \mathrm{yrs}$; PD duration $=12.3 \pm 2.7 \mathrm{yrs}$; duration of DBS $=25.6 \pm 21.2 \mathrm{mos}$, and UPDRS III speech score $=1.6 \pm 0.7$. STN subjects were studied off medication at their therapeutic DBS settings and again with their stimulators off, counter-balanced order.

Results: Vowel space was larger in the initiation phase compared to the midpoint for both the control and the STN subjects off stimulation. With stimulation on, however, the initial vowel space was significantly reduced to the area measured at the mid-point. For the three vowels, the acoustics were differentially affected, in accordance with expected effects of front versus back position in the vocal tract.

Conclusions: STN stimulation appears to constrain initial articulatory gestures for vowel production, raising the possibility that articulatory positions normally used in speech are similarly constrained.
\end{abstract}

Keywords: Deep brain stimulation, Parkinson's disease, speech, subthalamic nucleus, vowels

\footnotetext{
*Correspondence to: John J. Sidtis, Ph.D., Brain and Behavior Laboratory, Geriatrics Division The Nathan Kline Institute, 140
} 


\section{BACKGROUND}

Deep brain stimulation of the subthalamic nuclei (STN-DBS) is a widely used neurosurgical treatment for the levodopa responsive symptoms of Parkinson's disease (PD) with tens of thousands of individuals treated in this manner world-wide [1]. STN-DBS significantly improves tremor, rigidity, and bradykinesia, reduces on/off effects, and often allows for a lower dose of PD medication reducing the risk of drug-induced dyskinesias. While clinically effective, the physiological basis for this treatment is not understood [2-6].

In spite of concerns over adverse STN-DBS effects on speech, the objective evidence for this is inconsistent. In some instances, stimulation has had a demonstrably negative impact on speech $[7,8]$, but considering other studies, the nature of the speech changes associated with STN-DBS is far from clear (See Skodda, [9], for review). At the level of connected speech, Ahn et al. [10] found that long pauses, especially at non-linguistic boundaries, were more frequent with STN-DBS, and pausing during monologue speech was related to changes in cerebral blood flow during STN-DBS [11]. At the level of word production, relational timing (whereby stem vowel lengths decrease consistently with morphologically derived forms: stick, sticky, stickily; see Lehiste [12]) remains intact during STN-DBS [13]. In contrast, there is evidence that STN-DBS improves voice. Van Lancker Sidtis, et al. [14] found that the vocal signal-to noise improved during STN-DBS. Vowel production studies have reported an increased maximum phonation time [15-17] and greater stability in pitch and/or amplitude [8, 16-19], but this has not always been found [15]. Wang et al. [20] reported increased intensity in sustained vowel production following right but not left STN stimulation, but decreased articulatory accuracy following left but not right STN stimulation [21]. Lee et al. [22] found that STN-DBS reduced the aperiodicity in sustained production of /a/. Sauvageau et al. [23] reported increased vowel space with STN-DBS for CVCV syllables produced as part of a reading task.

While most of the reported acoustic changes in speech following STN-DBS have been inconsistently observed, clinically significant speech changes can also occur. Stimulation produces a three-dimensional field [2] and electrode placement and stimulation parameters can effect surrounding tissue [24], particularly the corticobulbar tract [25]. Stimulation of this tract is likely responsible for some of the side effects of STN-DBS including facial muscle contractions and dysarthria $[6,25]$. Stimulation of the fasciculus cerebellothalamicus has also been identified as a source of adverse effects on speech [26]. Excessive stimulation or inappropriate selection of stimulating electrode contacts can also produce abnormal speech in subjects who are otherwise unaffected at clinical settings [27, 28]. These extreme responses to STNDBS are likely not in the same class of phenomena as the previously noted changes in pausing, voice quality, and vowel characteristics as they approach or achieve the status of a clinically recognizable dysarthria. As such, they should probably be considered separately at this stage of our understanding of DBS effects on speech.

In contrast to the acoustic studies, several clinical studies have shown higher rates of speech problems following STN-DBS compared to medical therapy. As with the acoustic studies, however, the withingroup variability has been high $[29,30]$. Some of the post-STN-DBS changes were attributed to worsening of PD or pre-existing symptoms or corticobulbar side effects [30] and the most important predictive factor of post-STN-DBS speech intelligibility were pre-operative intelligibility, disease duration, and electrode placement [31].

The present study examined the effects of STNDBS on vowel space estimated during sustained vowel production. Vowel space is an important metric for speech as it reflects the configuration of the articulators [32] providing an estimate of the working range of articulator positions [33]. This configuration can be influenced by many factors including speaking rate and stress patterns [34-37], and phonological context [38]. Vowel space is also associated with intelligibility [39] and is altered by neurological disease [40]. It is reduced in amyotrophic lateral sclerosis, but less so in Parkinson's disease [36, 40-42]. Impairment in vowel articulation has been suggested as a marker of disease progression in PD [69]. Further, acoustic analysis of sustained vowel productions has been suggested as a tool for identifying STN-DBS overstimulation and fine tuning stimulation parameters [43].

Sustained vowel production provides a relatively stable opportunity to examine the acoustic characteristics of the vowel, which correspond to the articulatory gestures necessary to produce that vowel. The configuration of the vocal tract during vowel production reinforces acoustic energy produced by phonation into frequency bands referred to as formants, which can be changed by altering the shape of the vocal tract, thereby changing the identity of the 
vowel $[44,45]$. From the neurogenic perspective, the acoustic stability of sustained vowels involves basal ganglia regions likely affected by STN-DBS as well as cerebral and cerebellar regions [46]. When several different vowels are studied, their acoustic characteristics can be used to estimate a vowel space, measured as a function of the frequency values of the first (F1) and second (F2) formants.

While vowel space is typically obtained at the mid-portion of the vowel production to avoid onset and offset effects [22], the present study also examined vowel space during the initial $250 \mathrm{msec}$ of each production, enabling a contrast between possible STN-DBS effects on articulator positions at vowel initiation compared to a midpoint position where measurements are more typically made. Since movement initiation is one of the areas of difficulty in PD, it was believed that this initial component of vowel production might be particularly sensitive to the effects of STN-DBS. This hypothesis was further stimulated by an examination of all $\mathrm{F} 1$ and $\mathrm{F} 2$ data points produced by the PRAAT [47] analysis for the corner vowels $(/ \mathrm{i} /, / \mathrm{a} /, / \mathrm{u} /)$, which were obtained in vowel prolongation tasks as part of our speech production protocol. The following examination of vowel spaces at the initiation and midpoints of prolonged productions addressed the following questions: (1) does STN-DBS affect vowel space during sustained production? (2) does STN-DBS have a different effect at the initiation of vowel prolongation compared the vocalization midpoint?

\section{METHOD}

\section{Subjects}

Eight right-handed males with idiopathic PD and seven right-handed, neurologically normal, control male subjects participated in this study. All subjects provided informed consent for the study (Institutional Review Board, Nathan Kline Institute) consistent with the Declaration of Helsinki. Participants were native speakers of American English with the exception of one of the PD subjects, who reported that English was his native language in spite of the fact that he emigrated from Italy in his early adolescence. The mean age of the PD group was $57.5 \pm 4.4 \mathrm{yrs}$, which did not differ significantly from the mean age of the normal group $(57.4 \pm 11.1)$. The mean duration of PD was $13.4 \pm 3.3 \mathrm{yrs}$ and the mean duration of DBS therapy was $24 \pm 20$ months. The average Unified Parkinson's Disease Related Score (UPDRS) motor score was $34.3 \pm 16.7$, a moderate score that indicates some functional dependency [48] off both medication and STN-DBS. The average UPDRS speech rating was 1.6, consistent with the speech pathologist's characterization of mild dysarthria. The characteristics and treatment parameters of the subjects with STNDBS are presented in Table 1. The subjects with PD were selected based on right-handedness, native language, the absence of confounding neurological, psychiatric, or medical conditions and potentially confounding non-PD medications as well as their willingness to abstain from their anti-PD medication over-night, have their stimulators turned off for approximately five hours, and participate in a positron emission tomographic (PET) study that is part of a larger project. The presence or absence of speech complaints following STN-DBS treatment was not a selection criterion. All STN-DBS subjects had mild dysarthria as determined by an experienced, certified speech pathologist (D.S.), and none had more than a mild dysarthria. The normal controls met the same criteria with the exceptions related to PD treatment. PD subjects were tested in both the STN-DBS on and off conditions (order varied across participants)

Table 1

Characteristics of subjects with Parkinson's disease (PD) and bilateral deep brain stimulation of the subthalamic nuclei

\begin{tabular}{lccccccc}
\hline SID & AGE & PD YRS & DBS MOS & UPDRS SPEECH & LEVO & L.AMP & R.AMP \\
\hline 103 & 54 & 14 & 44 & 1 & 250 & 3.2 & 2.8 \\
104 & 57 & 17 & 27 & 2 & 400 & 3.0 & 3.0 \\
106 & 59 & 17 & 2 & 3 & 600 & 3.0 & 3.0 \\
107 & 62 & 17 & 12 & 2 & 600 & 2.5 & 2.6 \\
108 & 61 & 11 & 6 & 1 & 600 & 2.8 & 2.8 \\
109 & 49 & 9 & 36 & 2 & 300 & 2.6 & 2.5 \\
110 & 62 & 11 & 11 & 1 & 600 & 3.0 & 3.3 \\
111 & 56 & 11 & 400 & 3.0 & 3.0 \\
\hline
\end{tabular}

KEY: SID = subject identifier; AGE = in years; PD YRS = duration of PD; DBS MOS = duration of DBS therapy in months; UPDRS SPEECH = the speech rating in the UPDRS motor evaluation; $\mathrm{LEVO}=$ daily levodopa dose in $\mathrm{mg} ; \mathrm{L} . \mathrm{AMP}=$ amplitude of stimulation on the left side; R.AMP = amplitude of stimulation on the right side. All stimulation was at $185 \mathrm{~Hz}$ with a pulse width of 60 micro seconds. The UPDRS SPEECH ratings are 0 (normal), 1 (slight loss of expression, diction, and/or voice, 2 (monotone, slurred, but understandable), 3 (marked impairment but difficult to understand), and 4 (unintelligible). 
Table 2

F1 and F2 frequency values for /a/, /i/, and /u/ at measured during the initial 250 msec and at the middle point of production, for Individuals with Parkinson's disease with their deep-brain stimulators turned on at their normal therapeutic levels and turned off, and for normal control subjects

\begin{tabular}{|c|c|c|c|c|c|c|}
\hline \multirow[t]{2}{*}{ Condition } & \multicolumn{2}{|c|}{ /a/ } & \multicolumn{2}{|c|}{ /i/ } & \multicolumn{2}{|c|}{ /u/ } \\
\hline & Initial & Middle & Initial & Middle & Initial & Middle \\
\hline$O f f-F 1$ & $670.7(81)$ & $635.6(77)$ & $310.8(20)$ & $342.4(45)$ & 338.0 (39) & $360.9(56)$ \\
\hline$O n-F 1$ & $656.6(84)$ & $653.2(95)$ & 312.4 (34) & $329.0(30)$ & $332.5(39)$ & 332.5 (37) \\
\hline Nor $-F 1$ & 708.7 (76) & 649.7 (96) & 364.8 (123) & $306.2(24)$ & $335.4(28)$ & $329.4(42)$ \\
\hline$O f f-F 2$ & $1157.2(51)$ & $1090.5(150)$ & 2364.7 (215) & $2159.1(218)$ & 860.5 (113) & $851.4(153)$ \\
\hline$O n-F 2$ & $1103.6(58)$ & $1145.2(90)$ & $2112.9(258)$ & 2238.0 (209) & $896.2(140)$ & $813.4(133)$ \\
\hline Nor $-F 2$ & $1183.2(85)$ & $1252.0(148)$ & $2403.0(120)$ & $2239.0(183)$ & 957.1 (139) & $981.5(165)$ \\
\hline
\end{tabular}

having abstained from their PD medication since their last evening dose the night before the study. The speech samples were obtained the following mid-day after participation in an imaging study. In the STNDBS off condition, stimulation was off for at least three hours prior to the speech study.

\section{Procedure}

Sustained vowels were recorded as part of a protocol speech examination. Subjects were instructed to take a breath then produce the vowel /a/ in a steady fashion on that breath for as long as possible. This procedure was then repeated with the vowels /i/ and $/ \mathrm{u} /$. Vowel productions were digitally recorded for subsequent analyses. Recording were made using a Marantz Professional digital recorder (PMD660) and a Shure unidirectional head-worn dynamic microphone (SM10A). Simultaneous backup recordings were made with a separate PMD660 digital recorder and a boom-mounted AKG D5 microphone. All recordings were made in.wav format at a 48k sampling rate. Recordings were analyzed using PRAAT [47]. The formant frequencies of each sustained phonation were measured in one $250 \mathrm{msec}$ sample initiated at the onset of each vowel and a 2 sec sample taken from the midpoint of each vowel. In order to ensure accurate measurements across vowels and speakers, the automatic formant frequency values were visually compared to the simultaneous spectrogram display. To ensure agreement between the two methods, the upper frequency limit value for the formant listing program was adjusted to fit the height of the third formant, as estimated with a manual measurement of the spectrogram for each sample. The maximum frequency used ranged from $3000 \mathrm{~Hz}$ to $5000 \mathrm{~Hz}$.

\section{Measures}

The F1 and F2 values for the three vowels provided the $x, y$ coordinates of a triangle used to define a subject's vowel space. The area values for the initial and midpoint vowel space triangles were calculated for each subject. In addition to the vowel space measure, F1 and F2 values were also examined to determine the direction of changes in vowel space when they were observed. Table 2 contains the means (standard deviations in parentheses) for each vowel, each group, and each condition. The vowel space area was calculated with the following standard formula for determining the area of a triangle from $x, y$ coordinates with F1 values plotted against F2 values for each corner vowel:

$$
\begin{aligned}
& V S A=A B S((F 1 u *(F 2 i-F 2 a)+F 1 i * \\
& \quad(F 2 a-F 2 u)+F 1 a *(F 2 u-F 2 i)) / 2) \\
& \mathrm{VSA}=\text { vowel space area } \\
& \mathrm{ABS}=\text { absolute value } \\
& \mathrm{F} 1=\text { first formant } \\
& \mathrm{F} 2=\text { second formant }
\end{aligned}
$$

\section{Statistical analysis}

Initial and midpoint values of vowel spaces and $\mathrm{F} 1$ and $\mathrm{F} 2$ values were analyzed using mixed design repeated measures analyses of variance (ANOVAs) with a full-factorial model, and independent and paired $t$-tests as appropriate. Correlations were used to examine the relationships between variables (SPSS for PC version 7.5). Probability values ( $p$ ) less than 0.05 were considered significant.

\section{Results}

In order to rule out the possible development of jaw tremor during the sustained production [49] as confounding factor, for each vowel, the initial and mid-portion jitter (cycle-to-cycle fluctuations in frequency) and shimmer (cycle-to-cycle fluctuations in amplitude) measures were compared using a repeated measures ANOVA. None of the vowel productions contained significant main effects of STN-DBS condition (on vs. off) or position in the production (initial 
vs. midpoint), nor did they demonstrate an interaction between DBS status and position for either the jitter or shimmer measures.

\section{Vowel space}

The first set of analyses examined vowel space area obtained from the PD subjects with STN-DBS as an independent variable in a repeated measures ANOVA with position (initial vs. midpoint) and STN-DBS condition (on vs. off) treated as repeated measures. The normal control subjects were not included in this analysis. Neither position in the production (initial vs. midpoint) nor DBS condition (on vs. off) were significant as main effects, but these factors did interact $[F(1,7)=23.02 ; p=0.002]$. With STN-DBS off, the within subject comparison of position revealed that the initial vowel space was significantly greater than the subsequent midpoint vowel space $[t(7)=3.04$; $p=0.019]$. The within subject STN-DBS condition analysis revealed that initial vowel space with DBS off was also greater than the initial vowel space with STN-DBS on $[t(7)=2.52 ; p=0.04]$. In contrast, the within subject STN-DBS condition analysis revealed that when STN-DBS was on, the initial and midpoint vowel spaces did not differ. The results of this analysis are presented in Fig. 1. To summarize the within subject results, STN-DBS status interacted with vowel space position: (1) With STN-DBS off, initial vowel space was larger than midpoint vowel space. This was not the case when STN-DBS was on; (2) Initial vowel space was greater when STN-DBS was off compared to when STN-DBS was on.

The second set of mixed ANOVAs first compared the vowel space for the normal control subjects to the PD subjects with STN-DBS on, and in a separate analysis, to the PD subjects with STN-DBS off. Position in the vowel production (initial versus midpoint) was a repeated measure and group (normal control vs STN-DBS on in one analysis, normal control vs. STN-DBS off in the other) was a between-subjects factor. When the normal control vowel spaces were compared with those for STN-DBS off, there was an effect of position $[F(1,13)=16.8 ; p=0.001]$, with larger initial vowel spaces for both groups. There was no effect of group, and group and position did not interact (Fig. 1). As in the subjects with STN-DBS off, the initial normal vowel space was significantly greater than the stable vowel space $[t(6)=2.82$; $p=0.031]$.

In the comparison between the normal control group and the group with STN-DBS on, neither

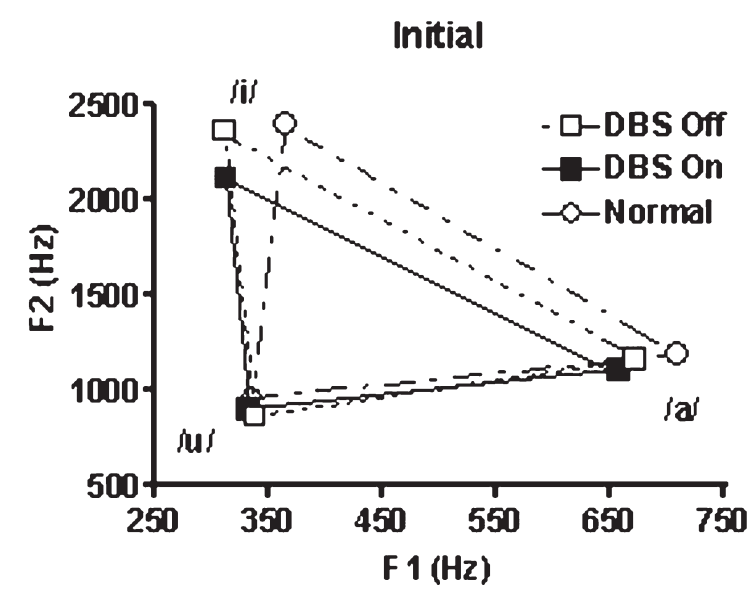

Midpoint

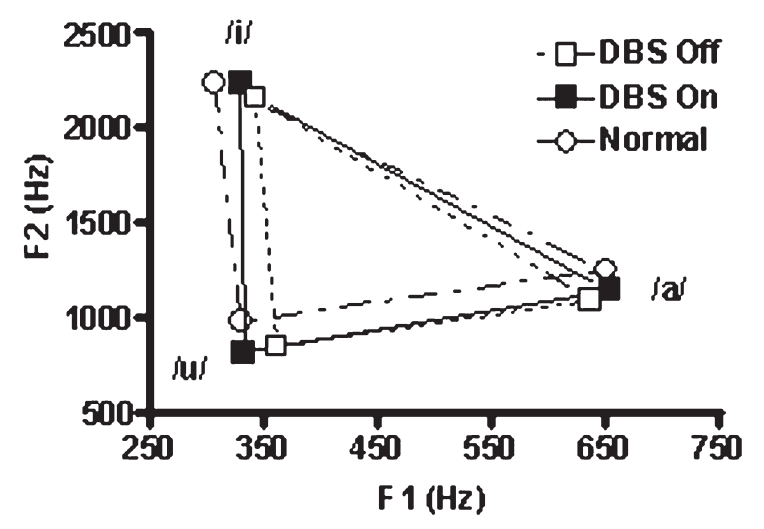

Fig. 1. Vowel space for $/ \mathrm{a} /, / \mathrm{i} /$, and $/ \mathrm{u} /$ representing the initial $250 \mathrm{msec}$ of the production and a $2 \mathrm{sec}$ portion at the production midpoint. The PD subjects were studied twice, once with STNDBS on and once with the stimulation off. Parkinson's medications were withheld for $12 \mathrm{hrs}$ prior to testing in both conditions. The age-matched normal subjects were studied once.

position nor group (i.e., STN-DBS on) significantly influenced vowel space, but these factors did interact $[F(1,13)=9.72 ; p=0.008]$. The initial normal control vowel spaces were larger than the initial STNDBS on vowel spaces. The results of the between groups analyses are as follows: (1) Both the normal subjects and those with PD with STN-DBS turned off had larger vowel spaces at initiation compared to midpoint; (2) When the normal group was compared to the STN-DBS on group, only the normal group demonstrated a larger initial vowel space.

In order to examine the possible relationships between the vowel space measures and clinical characteristics of the STD-DBS subjects, several 
correlations were performed. The size of initial vowel space was not correlated with age in either of the STN-DBS conditions. However, the size of the initial vowel space was negatively correlated with duration of PD in both the STN-DBS off $[r(8)=-0.754$; $p=0.03]$ and STN-DBS on $[r(8)=-0.748 ; p=0.03]$ conditions. This relationship indicated that initial vowel space declined as the duration of PD increased. In addition, vowel spaces in these two conditions were positively correlated $[r(8)=0.717 ; p=0.045]$ with each other, suggesting that STN-DBS was attenuating vowel space rather than creating novel vowel spaces. The UPDRS Speech score was marginally correlated with the duration of $\mathrm{PD}(r=0.71 ; p=0.05)$ indicating a decline in speech rating with an increase in the duration of PD. The UPDRS Speech score was negatively correlated with both the initial vowel space $(r=-0.76 ; p=0.029)$ and the vowel space at midpoint $(r=-0.81 ; p=0.014)$ indicating that vowel space reduction was associated with poorer speech ratings. Although the range of the duration of DBS therapy was large in this sample, it was not significantly correlated with the change in vowel space.

\section{First and second formants by vowel}

As vowel space is a composite measure of two formants measured for the production of three corner vowels, an examination of the formant values for each vowel might provide some indication of the major loci of the vowel space effects. During the production of the low vowel, /a/, there were significant interactions between position and STN-DBS status for F1 $[F(1,7)$ $=8.55 ; p=0.022]$ and $\mathrm{F} 2[F(1,7)=7.55 ; p=0.029]$. This interaction was only present for $\mathrm{F} 2$ during the



Fig. 2. Average F2 values across vowels for the three study conditions. Asterisks indicate significant differences between groups $(p<0.05)$ production of $/ \mathrm{i} /[F(1,7)=6.71 ; p=0.036]$. For $/ \mathrm{i} /$, initial F2 was reduced by $11 \%$ in the STN-DBS on condition $[t(7)=2.47 ; p=0.04]$. In contrast, for the back vowel/u/, F1 and F2 changes in association with DBS on were not significant, suggesting that STNDBS does not have a uniform effect on all articulatory gestures. Figure 2 depicts the means and standard errors of F2 across vowels for the three conditions.

\section{DISCUSSION}

Both normal control and PD subjects with their STN-DBS turned off initiated sustained vowel productions with a larger vowel space than they used at the midpoint of their production. This effect was not present when the same PD subjects had their STN-DBS turned on. With STN-DBS on, the initial vowel space was smaller, but did not decrease further at the midpoint position. The consistency of the midpoint vowel space area measurements across both subject groups and all three conditions, as well as the consistency between the initial vowel space measurements for the normal control subjects and the PD subjects with the DBS turned off, strongly suggest that these measurements were stable, reliable, and representative. Rather than creating an aberrant vowel space, STN-DBS appears to have attenuated the vowel space normally available to the PD and normal control subjects at the point of vowel initiation, thereby restricting the normally available articulatory range. This effect was not uniform across vowels, however, as it was not observed for the back vowel/u/, which is produced by upward and rostral movement of the back of the tongue.

Speech motor control depends on the planning, initiation, and execution of articulatory sequences and involves detailed coordination with laryngeal and respiratory processes. The present results may bear on the effects of STN-DBS at the initiation of articulatory gestures, with implications for planning and coordination as well. The reduction of initial vowel space associated with STN-DBS on suggests that at least some articulatory gestures are constrained at their initiation stage. As seen in the present study, such constraints were not sufficient to grossly distort the target vowel. As noted previously, PD itself reduces vowel space, at least in some speech modes, so STN-DBS is further constraining an already affected articulatory system. This may occur because STN-DBS alters the internal mapping of the articulators, the afferent feedback about their states, 
the efferent commands to create the gestures, the motor tone of specific articulators, the coordination of articulatory, laryngeal, and respiratory components, or any combination of the above. Such a situation could also account for motor control problems that result in the abnormal pausing [10] and for the case reports of dysfluency $[14,51,52]$ observed with STNDBS.

Constraints on articulatory control are also likely to interact with the type of speech produced, as the quality of speech in PD is a function of the speech task, whether STN-DBS is involved or not [14, 52-57]. Speaking spontaneously requires more planning than reading or repetition, as these latter speaking modes have an external model. In PD, spontaneous speech is less intelligible than speech that is repeated or read $[54,55,58]$. Task effects may also play a role in studies of vowel space. Skodda et al. [59] found that vowel space was reduced in PD and declined with disease progression based on an analysis of read speech. However, Sauvageau et al. [23] reported that vowel space derived from CVCV syllables embedded in read sentences increased with STN-DBS. In the present study, vowel spaces in the STN-DBS on and off conditions were positively correlated with each other, and both were reduced as the duration of PD increased. The observed reduction with disease progression is consistent with Skodda's findings, but overall vowel space was not reduced in the subjects with PD in the present study. The different findings with respect to the effects of PD and STNDBS on vowel space when the Skodda and Savageau results are compared to the present study may reflect several differences: Both Skodda and Savageau analyzed read material and their vowel productions were shorter than the sustained productions. It may also be the case that their subjects were more severely affected by PD than those in the present study. In any case, it is clear that the effects of speaking mode have a significant impact on the quality of speech in PD and will have to be considered in an accurate characterization of the impact of PD and STN-DBS on speech.

In addition to the linguistic and phonological constraints on speech motor control, more general effects of STN-DBS need to be considered. The absence of an STN-DBS effect on the back vowel $/ \mathrm{u} /$, which involves upward and rostral movement of the back of the tongue, hints that STN-DBS may have differential impact on different muscle groups (e.g., the muscles of the tongue). This invites an interesting parallel with swallowing. Like speech, there is a dissociation between subjective reports and the paucity of supporting objective findings regarding STN-DBS's impact on swallowing. Unlike speech, however, subjects have reported improved swallowing following STN-DBS [60-63]. In swallowing studies, small but significant improvements were reported for pharyngeal transit time but not for maximum hyoid bone excursion with STN-DBS on compared to off conditions [63]. Recent reports on the effects of STN-DBS on swallowing have also associated this therapy with changes in tongue function $[64,65]$. The present results for vowel space in speech and for pharyngeal transit time and tongue function in swallowing suggest that changes in motor function may result in disproportionate subjective experiences regarding movements involving articulatory structures. The variability in self-report of speech difficulties has been shown to be greater in PD subjects who have been treated with STN-DBS compared to those treated medically [66]. Such variability in self report measures following STN-DBS mirrors the variability in acoustic results across studies of STNDBS effects, and may indicate that not all changes in speech-motor control yield comparable subjective complaints.

This study, together with the existing literature, provides an emerging picture of the effects of STNDBS on speech. With the exception of excessive stimulation, inappropriate stimulation site, or surgical complication, the objective measurements of speech change following STN-DBS have demonstrated inconsistent results. The most consistent findings are in the domain of voice, in which some degree of improved function has been reported. These results, obtained with utterances of varying lengths in different production modes, as well as with sustained vocal productions, likely reflect, at least in part, improved glottal and sub-glottal control as demonstrated in the work of Hammer et al. [67, 68], who reported increased intraoral pressure and increased velopharyngeal closure associated with STN-DBS.

It may be the case that STN-DBS effects can improve glottal and sub-glottal support for speech, but that it can also introduce fine-grained alterations of articulatory gestures, predictably at the initiation of speech acts. It can be hypothesized that improved phonation may tax an articulatory control system already weakened by PD and further compromised by an alteration of the basal ganglia's role in motor control resulting from STN-DBS. This combination of pathophysiological and iatrogenic changes could exacerbate problems with motor initiation, pausing 
during speech, fluency, and intelligibility. The combination could also produce a subjective sense, in some individuals, that speech is more difficult after treatment by STN-DBS.

The present results demonstrate that STN-DBS constrains vowel space at the onset of prolonged vowel production. Both the normal control group and the subjects with Parkinson's disease who had their STN-DBS turned off initiated their productions with a larger vowel space, which was reduced by the midpoint of the production. However, when STN-DBS was turned on, initial vowel spaces were reduced and did not change from initial to midpoint values. These results suggest that STN-DBS can constrain articulatory gestures, which may contribute to the subjective experience that speech has become more difficult after STN-DBS, and when severe enough, contribute to dysfluent speech. It should also be noted that STNDBS is not a unitary entity. The effects of this therapy are a function of surgical technique, programming, medication, disease state, clinical features, and individual differences in patients and their neuroanatomy. It is unlikely that there is a single answer to the question of how STN-DBS affects speech. However, the results of this study add to the existing literature suggesting that acoustic changes in voice may be a valuable tool in assessing speech changes. From a speech-science perspective, these results suggest that an examination of performance at the initiation of speech acts may provide insights into the consequences of PD and STN-DBS.

\section{ACKNOWLEDGMENTS}

The contributions of the Brain and Behavior Laboratory assistants in conducting the acoustic analyses is greatly appreciated. This work was supported by a grant from the National Institute of Deafness and Communicative Disorders (R01 DC 007658).

\section{CONFLICT OF INTEREST}

The authors have no conflict of interest to support.

\section{REFERENCES}

[1] Volkmann J (2007) Deep brain stimulation for Parkinson's disease. Parkinsonism Relat Disord, 13, S462-S465.

[2] McIntyre CC, Savasta M, Walter BL, \& Vitek JL (2004) How does deep brain stimulation work? Present understanding and future questions. J Clin Neurophysiol, 21, 40-50.

[3] Perlmutter JS, \& Mink JW (2006) Deep brain stimulation. Annu Rev Neurosci, 29, 229-257.
[4] Montgomery EB (2007) Basal ganglia physiology and pathophysiology: A reappraisal. Parkinsonism Relat Disord, 13, 455-465.

[5] Vitek JL (2008) Deep brain stimulation: How does it work? Cleve Clin J Med, 75, S59-S65.

[6] Benabid AL, Chabardes S, Mitrofanis J, \& Pollak P (2009) Deep brain stimulation of the subthalamic nucleus for the treatment of Parkinson's disease. Lancet Neurol, 8, 67-81.

[7] Kleiner-Fisman G, Herzog J, Fisman DN, Tamma F, Lyons KE, Pahwa R, Lang AE, \& Deuschl G (2006) Subthalamic nucleus deep brain stimulation: Summary and meta-analysis of outcomes. Mov Disord, 21, S290-S304.

[8] Klostermann F, Ehlen F, Vesper J, Nubel K, Gross M, Marzinzik F, Curio G, \& Sappok T (2009) Effects of subthalamic deep brain stimulation on dysarthrophonia in Parkinson's disease. J Neurol Neurosurg Psychiatry, 79, 522-529.

[9] Skodda S (2012) Effects of deep brain stimulation on speech performance in Parkinson's disease. Parkinsons Dis, 2012, 850596.

[10] Ahn JS, Van Lancker Sidtis D, \& Sidtis JJ (2014) Effects of deep brain stimulation on pausing during spontaneous speech in Parkinson's disease. J Med Speech Lang Pathol 21, 179-186.

[11] Sidtis JJ, Tagliati M, Alterman R, Van Lancker Sidtis D, Dhawan V, \& Eidelberg D (2012) Therapeutic high frequency stimulation of the subthalamic nucleus in Parkinson's produces global increases cerebral blood flow. J Cereb Blood Flow Metab, 32, 41-49.

[12] Lehiste I (1972) The timing of utterances and linguistic boundaries. J Acoust Soc Am, 51, 2018-2024.

[13] Sidtis JJ, \& Van Lancker Sidtis D (2013) Preservation of relational timing in speech of persons with Parkinson's disease with and without deep brain stimulation. J Med Speech Lang Pathol, 20, 140-151.

[14] Van Lancker Sidtis D, Rogers T, Godier V, Tagliati M, \& Sidtis JJ (2010) Voice and fluency changes as a function of speech task and deep brain stimulation. J Speech Lang Hear Res, 53, 1167-1177.

[15] Sung JE, Kim H, Kim HS, Oh S-H, Hong J-M, \& Lee MS (2004) Effects of subthalamic nucleus deep brain stimulation on the phonation and articulation of patients with Parkinson's disease. J Korean Neurol Assoc, 22, 472-477.

[16] Gentil M, Chauvin P, Pinto S, Pollak P, \& Benabid AL (2001) Effect of bilateral stimulation of the subthalamic nucleus on Parkinsonian voice. Brain Lang, 78, 233-238.

[17] Gentil M, Pinto S, Pollak P, \& Benabid A-L (2003) Effect of bilateral stimulation of the subthalamic nucleus on parkinsonian dysarthria. Brain Lang, 85, 190-196.

[18] D'Alatri L, Paludetti G, Contarino MF, Galla S, Marchese MR, \& Bentivoglio AR (2008) Effects of bilateral subthalamic nucleus stimulation and medication on Parkinsonian speech impairment. $J$ Voice, 22, 365-372.

[19] Hoffman-Ruddy B, Schulz G, Vitek J, \& Evatt M (2001) A preliminary study of the effects of subthalamic nucleus (STN) deep brain stimulation (DBS) on voice and speech characteristics in Parkinson's disease (PD). Clin Linguist Phon, 15, 97-101.

[20] Wang E, Metman LV, Bakay R, Arzbaecher J, \& Bernard B (2003) The effect of unilateral electrostimulation of the subthalamic nucleus on respiratory/phonatory subsystems of speech production in Parkinson's disease-a preliminary report. Clin Linguist Phon, 17, 283-289.

[21] Wang EQ, Metman LV, Bakay RAE, Arzbaecher J, Bernard B, \& Corcos DM (2006) Hemisphere-specific effects of subthalamic nucleus deep brain stimulation on speaking rate 
and articulatory accuracy of syllable repetitions in Parkinson's disease. J Med Speech Lang Pathol, 14, 323-334.

[22] Lee VS, Zhou XP, Rahn DA, Wang EQ, \& Jiang JJ (2008) Perturbation and nonlinear analysis of acoustic phonatory signal in Parkinsonian patients receiving deep brain stimulation. J Commun Disord, 41, 485-500.

[23] Sauvageau VM, Macoir J, Langlois M, Prud'Homme M, Cantin L, \& Roy J-P (2014) Changes in vowel articulation with subthalamic nucleus deep brain stimulation in dysarthric speakers with Parkinson's disease. Parkinsons Dis, 2014, 487035.

[24] Maks CB, Butson CR, Walter BL, Vitek JL, \& McIntyre CC (2009) Deep brain stimulation activation volumes and their association with neurophysiological mapping and therapeutic outcomes. J Neurol Neurosurg Psychiatry, 80, 659-666

[25] Tommasi G, Krack P, Fraix F, Le Bas J-F, Chabardes S, Benabid A-L, \& Pollak P (2008) Pyramidal tract side effects induced by deep brain stimulation of the subthalamic nucleus. J Neurol Neurosurg Psychiatry, 79, 813-819.

[26] Åström M, Tripoliti E, Hariz MI, Zrinzo LU, MartinezTorres I, Limousin P, \& Wårdell K (2010) Patient-specific model-based investigation of speech intelligibility and movement during deep brain stimulation. Stereotact Funct Neurosurg, 88, 224-233.

[27] Törnqvist AL, Schalén L, \& Rehncrona S (2005) Effects of different electrical parameter settings on the intelligibility of speech in patients with Parkinson's disease treated with subthalamic deep brain stimulation. Mov Disord, 20, 416-423.

[28] Tripoliti E, Zrinzo L, Martinez-Torres I, Tisch S, Frost E, Borrell E, Hariz MI, \& Limousin P (2008) Effects of contact location and voltage amplitude on speech and movement in bilateral subthalamic nucleus deep brain stimulation. Mov Disord, 23, 2377-2383.

[29] Tripoliti E, Zrinzo L, Martinez-Torres I, Frost E, Pinto S, Foltynie T, Holl E, Petersen E, Roughton M, Hariz MI, \& Limousin P (2011) Effects of subthalamic stimulation on speech of consecutive patients with Parkinson disease. Neurology, 76, 80-86.

[30] Tsuboi T, Watanabe H, Tanaka Y, Ohdake R, Yoneyama N, Hara K, Nakamura R, Watanabe H, Senda J, Atsuta N, Ito M, Hirayama M, Yamamoto M, Fujimoto Y, Kajita Y, Wakabayashi T, \& Sobue G (2015) Distinct phenotypes of speech and voice disorders in Parkinson's disease after subthalamic nucleus deep brain stimulation. $J$ Neurol Neurosurg Psychiatry, 86, 856-864.

[31] Tripoliti E, Limousin P, Foltynie T, Candelario J, AvelesOlmos I, Hariz MI, \& Zrinzo L (2014) Predictive factors of speech intelligibility following subthalamic nucleus stimulation in consecutive patients with Parkinson's disease. Mov Dis, 29, 532-538.

[32] Ladefoged P, \& Disner SF (2012) Vowels and Consonants. Wiley-Blackwell, Malden, MA.

[33] Karlsson F, \& van Doorn J (2012) Vowel formant dispersion as a measure of articulation proficiency. J Acoust Soc Am, 132, 2633-2641.

[34] Miller JL (1981) Effects of speaking rate on segmental distinctions. In Perspectives on the Study of Speech, Eimas PD, Miller JL, eds. Erlbaum, Hillsdale NJ, pp. 39-74.

[35] Fourakis M (1991) Tempo, stress, and vowel reduction in American English. J Acoust Soc Am, 90, 1816-1827.

[36] McRae PA, Tjaden K, \& Schoonings B (2002) Acoustic and perceptual consequences of articulatory rate change in Parkinson disease. J Speech Lang Hear Res, 45, 35-50.
[37] Tsao Y-C, Weismer G, \& Iqbal K (2006) The effect of intertalker speech rate variation on acoustic vowel space. $J$ Acoust Soc Am, 119, 1074-1082.

[38] Munson B, \& Solomon NP (2004) The effect of phonological neighborhood density on vowel articulation. J Speech Lang Hear Res, 47, 1048-1058.

[39] Ferguson SH, \& Kewley-Port D (2007) Talker differences in clear and conversational speech: Acoustic characteristics of vowels. J Speech Lang Hear Res, 50, 1241-1255.

[40] Weismer G, Jeng J-Y, Laures JS, \& Kent RD (2001) Acoustic and intelligibility characteristics of sentence production in neurogenic speech disorders. Folia Phoniatr Logop, 53, $1-18$.

[41] Turner GS, Tjaden K, \& Weismer G (1995) The influence of speaking rate on vowel intelligibility for individuals with amyotrophic lateral sclerosis. J Speech Hear Res, 38, 10011013.

[42] Tjaden K, Rivera D, Wilding G, \& Turner GS (2005) Characteristics of the lax vowel space in dysarthria. $J$ Speech Lang Hear Res, 48, 554-566.

[43] Valálik I, Smehák G, Bognár L, \& Csókay A (2011) Voice acoustic changes during bilateral subthalamic stimulation in patients with Parkinson's disease. Clin Neurol Neurosurg, 113, 188-195.

[44] Klatt DH (1982) Prediction of perceived phonetic distance from critical-band spectra: A first step. IEEE ICASSP, 12781281.

[45] Hillenbrand JM, Houde RA, \& Gayvert RT (2006) Speech perception based on spectral peaks versus spectral shapes. $J$ Acoust Soc Am, 119, 4041-4054.

[46] Sidtis JJ (2015) Functional connectivity associated with acoustic stability during vowel production: Implications for vocal-motor control. Brain Connect, 5, 115-125.

[47] Boersma P, \& Weenink D (2009) Praat: Doing phonetics by computer (Version 51 11) [Computer program] Retrieved July 19, 2009, from http://www praat org/

[48] Shulman LM, Gruber-Baldini AL, Anderson KE, Fishman PS, Reich SG, \& Weiner WJ (2010) The clinically important difference on the Unified Parkinson's Disease Rating Scale. Arch Neurol, 67, 64-70.

[49] Boutsen F, Wu D, \& Stevens A (2008) Physiologic jaw tremor under different deep brain stimulation conditions in patients with Parkinson's disease. Paper presented at the Fourteenth Biennial Conference on Motor Speech. Monterey, California.

[50] Moretti R, Torre P, Antonello RM, Capus L, Gioulis M, Marsala SZ, Cazzato G, \& Bava A (2003) Speech initiation hesitation following subthalamic nucleus stimulation in a patient with Parkinson's disease. Eur Neurol, 49, 251-253.

[51] Toft M, \& Dietrichs E (2011) Aggravated stuttering following subthalamic deep brain stimulation in Parkinson's disease - two cases. BMC Neurol, 11, 44.

[52] Van Lancker Sidtis D, Cameron K, \& Sidtis JJ (2010) Dramatic effects of speech task on motor and linguistic planning in severely dysfluent parkinsonian speech. Clin Linguist Phon, 26, 695-711.

[53] Dromey C, Kumar R, Lang AE, \& Lozano AM (2000) An investigation of the effects of subthalamic nucleus stimulation on acoustic measures of voice. Mov Disord, 15 , 1132-1183.

[54] Kempler D, \& Van Lancker D (2002) The effect of speech task on intelligibility in dysarthria: Case study of Parkinson's disease. Brain Lang, 80, 449-464.

[55] Van Lancker Sidtis D, Cameron K, Bonura L, \& Sidtis JJ (2012) Speech intelligibility by listening in Parkinson 
speech with and without deep brain stimulation: Task effects. J Neurolinguistics, 25, 121-132.

[56] Karlsson F, Olofsson K, Blomstedt P, Linder P, \& van Doorn J (2013) Pitch variability in patients with Parkinson's disease: Effects of deep brain stimulation of caudal zona incerta and subthalamic nucleus. J Speech Lang Hear Res, 56, 150158.

[57] Bridges KA, Van Lancker Sidtis D, \& Sidtis JJ (2013) The role of subcortical structures in recited speech: Studies in Parkinson's disease. J Neurolinguistics, 26, 591-601.

[58] Canter GJ, \& Van Lancker D (1985) Disturbances of temporal organization of speech following bilateral thalamic surgery in a patient with Parkinson's disease. J Commun Disord, 18, 329-349.

[59] Skodda S, Grönheit W, \& Schlegel U (2012) Impairment of vowel articulation as a possible marker of disease progression in Parkinson's disease. PLoS One, 7, e32132.

[60] Silbergleit AK, LeWitt P, Junn F, Schultz LR, Collins D, Beardsley T, Hubert M, Trosch R, \& Schwalb JM (2012) Comparison of dysphagia before and after deep brain stimulation in Parkinson's disease. Mov Disord, 27, 1763-1768.

[61] Kulneff L, Sundstedt S, Olofsson K, van Doorn J, Linder J, Nordh E, \& Blomstedt P (2013) Deep brain stimulation effects on swallowing function in Parkinson's disease. Acta Neurol Scand, 127, 329-336.

[62] Troche MS, Brandimore AE, Foote KD, \& Okun MS (2013) Swallowing and deep brain stimulation in Parkinson's disease: A systematic review. Parkinsonism Relat Disord, 19, 783-788.
[63] Ciucci MR, Barkmeier-Kraemer JM, \& Sherman SJ (2008) Subthalamic nucleus deep brain stimulation improves deglutition in Parkinson's disease. Mov Disord, 23, 676-683.

[64] Kitashima A, Umemoto G, Tsuboi Y, Higuchi M, Baba Y, \& Kikuta T (2013) Effects of subthalamic nucleus deep brain stimulation on the swallowing function of patients with Parkinson's disease. Parkinsonism Relat Disord, 19, 480-482.

[65] Termsarasab P, \& Winona T (2015) Deep brain stimulation and orophartngeal dysfunction in Parkinson's disease: A case report. Parkinsonism Relat Disord, 21, 79-80.

[66] Frost E, Tripoliti E, Hariz MI, Pring T, \& Limousin P (2010) Self-perception of speech changes in patients with Parkinson's disease following deep brain stimulation of the subthalamic nucleus. Int J Speech Lang Path, 12, 399-404.

[67] Hammer MJ, Barlow SM, Lyons KE, \& Pahwa R (2010) Subthalamic nucleus deep brain stimulation changes speech respiratory and laryngeal control in Parkinson's disease. J Neurol, 257, 1692-1702.

[68] Hammer MJ, Barlow SM, Lyons KE, \& Pahwa R (2011) Subthalamic nucleus deep brain stimulation changes velopharyngeal control in Parkinson's disease. J Commun Disord, 44, 37-48.

[69] Skodda S, Grönheit W, \& Schlegel U (2012) Impairment of vowel articulation as a possible marker of disease progression in Parkinson's disease. PLoS One, 7, e32132. 